\title{
Understanding the Effect of Gloves on Hand-Arm Vibrations in Road Cycling ${ }^{\dagger}$
}

\author{
Giuseppe Sanseverino ${ }^{1}$, Stefan Schwanitz ${ }^{2, *}$, Dominik Krumm ${ }^{2}$, Stephan Odenwald ${ }^{2}$ \\ and Antonio Lanzotti ${ }^{1}$ \\ 1 Fraunhofer JL IDEAS, Department of Industrial Engineering, University of Naples Federico II, \\ 80125 Naples, Italy; sanseverinogiuseppe@outlook.it (G.S.); antonio.lanzotti@unina.it (A.L.) \\ 2 Department of Sports Equipment and Technology, Chemnitz University of Technology, 09107 Chemnitz, \\ Germany; dominik.krumm@mb.tu-chemnitz.de (D.K.); stephan.odenwald@mb.tu-chemnitz.de (S.O.) \\ * Correspondence: stefan.schwanitz@mb.tu-chemnitz.de; Tel.: +49-371-531-36657 \\ + Presented at the 13th conference of the International Sports Engineering Association, Online, \\ 22-26 June 2020.
}

Published: 15 June 2020

\begin{abstract}
The aim of this research was to study the effects of cycling gloves on hand-arm vibrations in realistic load scenarios. A test has been performed in the laboratory, a road bicycle handlebar was mounted to the hydraulic cylinder of a universal testing machine, and the bicycle was fixed on an indoor trainer. Tests were executed for three different hand sizes (small, medium, large), three different frequency ranges $(15-25,35-45,85-95 \mathrm{~Hz})$, with two different types of gloves (gel-padded; non-padded) and without gloves. The amplitudes and each frequency bands were obtained from a previous field test. Hand-arm vibrations were quantified by means of root mean square values of the frequency-weighted accelerations measured at the subject's wrist joint. Analysis of variance (ANOVA) showed no significant effect of gloves in reducing vibration transmissibility.
\end{abstract}

Keywords: bioengineering; sports equipment; road cycling; vibration transmission; laboratory test; field test; design of experiments

\section{Introduction}

Vibrations induced from the road profile to the handlebar of a road bicycle affect the cyclist's hand-arm system. In certain conditions of magnitude and frequency, vibrations can lead to injuries and disorders. In the case of a hand-arm system, there are four types of disorders: vascular disorders, neurological disorders, carpal tunnel syndrome, and repetitive strain injuries [1].

Numerous studies involving hand-arm vibrations (HAV) in road cycling have been carried out, both in the field and in laboratories, showing varying viewpoints on this complex topic. Some were focused on the acquisition of vibrations in the field and how to properly reproduce these in the laboratory. Others dealt with the factors that could affect the transmission of vibrations to the human body (e.g. hand position, cyclist's mass, speed, and tire pressure) [2,3].

Several investigations related to the effect of working gloves on hand-arm vibrations transmission have been reported in the literature [4], but few studies go deep into the analysis of the effect of different cycling gloves on vibrations transmission to the hand-arm system. Drouet et al. [5] studied the effect of cycling gloves and bar tape on vibrations transmission with only one type of gloves and one subject. This study showed a significant effect of bar tape in reducing the transmitted power and energy through the handlebar, but no significant effect of gloves. The aim of this research was to investigate the extent of hand-arm vibrations in road cycling and understand if cycling gloves do affect HAV transmission in realistic load scenarios or not. The study was carried out in the 
laboratory to quantify the transmissibility of different types of cycling gloves and to understand their effect on vibrations transmission.

\section{Materials and Methods}

\subsection{Objects of Investigation and Subjects}

Two different kinds of cycling gloves (Figure 1) were used as objects of investigation. Nonpadded gloves (P.R.O. Aero Gloves, PEARL iZUMi USA, Inc., Louisville, CO, USA) and gel-padded gloves (Men's Elite Gel Glove, PEARL iZUMi USA, Inc., Louisville, CO, USA) were purchased in three different sizes (small, medium, large). In addition to non-padded gloves (NPG) and padded gloves (PG), bare hands (no gloves, NG) were set as the third hand-equipment condition.

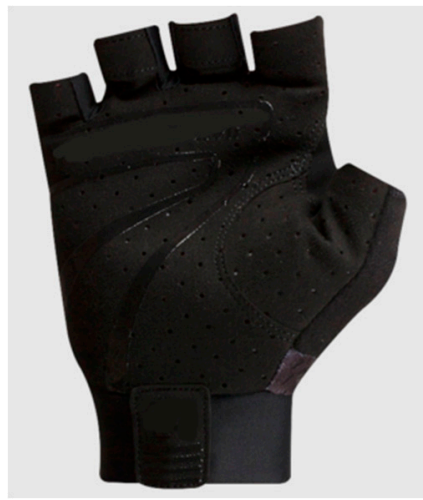

(a)

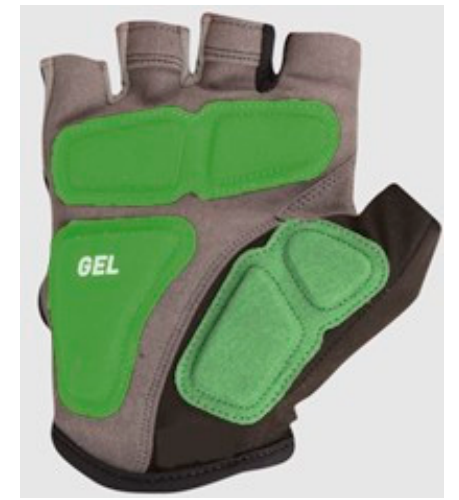

(b)

Figure 1. Cycling gloves used for the test. (a) Non-padded gloves; (b) gel-padded gloves (the gel-pads are highlighted in green).

Six subjects volunteered in this study. All of them were recreational cyclists and free from injuries of the hand-arm system. They were selected to match the given glove sizes regarding their hand circumferences (S: 18.5-20.3 cm, M 20.3-22.2 cm, L: 22.2-24.0 cm). All participants provided written informed consent. The study was in accordance with the Declaration of Helsinki.

\subsection{Apparatus}

The laboratory-based test setup (Figure 2) consisted of a road bike with a carbon frame (prototype, Martec Industrial Corp., Chu-Nan Town, Taiwan). The bicycle was fixed to both a bicycle treadmill (Flow T2200, Tacx B.V., Wassenaar, The Netherlands) and the base frame of a hydraulic testing machine (HC 10, ZwickRoell GmbH \& Co. KG, Ulm, Germany). The handlebar (TAQ-33 HB12, width $420 \mathrm{~mm}$, BICO Zweirad Marketing GmbH, Verl, Germany) was disconnected from the bicycle and fixed to the vertical actuator of the testing machine. Seat height, as well as stack and reach of the bicycle, were adjustable to fit the individual subject's preferences.

To quantify the extent of the vibrations, two triaxial ICP ${ }^{\circledR}$ accelerometers (354A04, PCB Piezotronics, Depew, NY, USA) were positioned at the right bar ending (ahandlebar) and the subject's right wrist (ulnar styloid process, awrist). The measurement system comprised two four-channel signal conditioners (482C05, PCB Piezotronics, Depew, NY, USA) and a data acquisition system (CS7008, imc Messsysteme $\mathrm{GmbH}$, Berlin, Germany). The sampling rate was set to $10 \mathrm{kHz}$. 


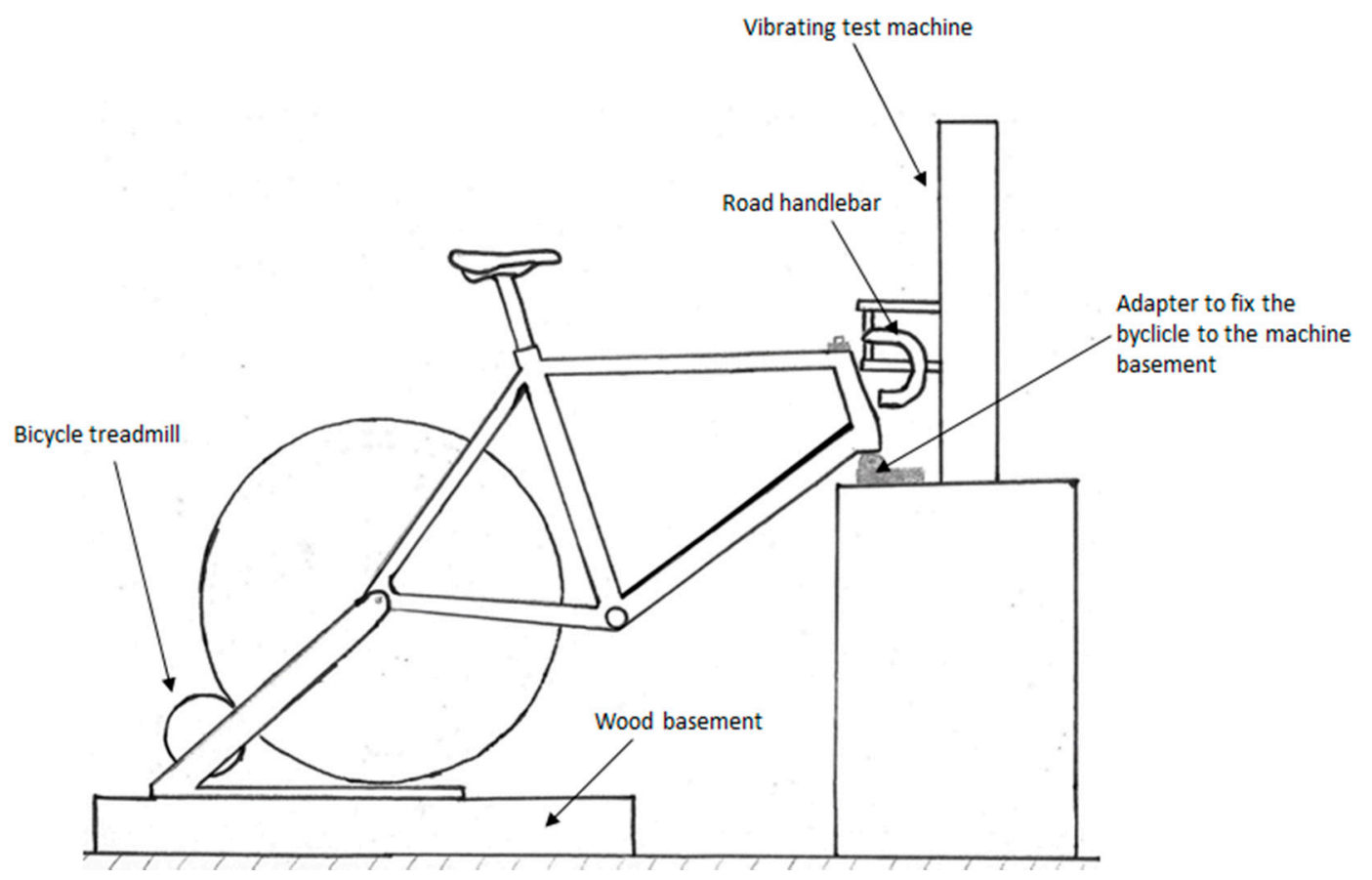

Figure 2. Laboratory test set-up.

\subsection{Test Protocol}

Subjects were asked to pedal with constant cadence and resistance of the bicycle treadmill. Within a warm-up period of five minutes, the gearing of the road bike was adjusted to make the subjects feel comfortable while pedaling. All vibration excitation tests were performed by holding the handlebar in the drops with self-selected grip forces with either of three hand-equipment conditions NG, NPG, and PG.

The test machine performed sinusoidal oscillations in the vertical axis. Three load conditions were derived from field test data and implemented in the test protocol. The actuator vibrated with an amplitude (d) of $0.98 \mathrm{~mm}$ and a frequency (f) of $20 \mathrm{~Hz}$ for load condition A, load condition B $(\mathrm{d}=0.71 \mathrm{~mm} ; \mathrm{f}=40 \mathrm{~Hz})$ and load condition $\mathrm{C}(\mathrm{d}=0.08 \mathrm{~mm} ; \mathrm{f}=90 \mathrm{~Hz})$. Those conditions mimic natural cycling on cobblestones by means of dominant frequencies and vibrational energy.

Each trial lasted $60 \mathrm{~s}$, followed by an active rest period of $60 \mathrm{~s}$. Five repeated measurements were obtained for each hand-equipment-load-condition combination. For each hand-equipment condition, all load conditions were tested subsequently in randomized order. During the changeover of hand equipment condition, subjects stopped pedaling for $120 \mathrm{~s}$. Overall, 45 trials were performed by each subject.

\subsection{Data Processing}

The software package DIAdem 2017 Professional (National Instruments Inc., Austin, TX, USA) was used to process the data acquired in 270 trials. The three axes of both accelerometers were filtered applying the evaluation filter $\mathrm{W}_{\mathrm{h}}$ to obtain the frequency-weighted accelerations [6]. The root mean squares (RMS) of the resultants were calculated in order to quantify the total vibration values av,wrist and $\mathrm{av}$,handlebar. The transmissibility was calculated as the ratio of the total vibration at the wrist and the handlebar.

$$
\text { Transmissibility }=\frac{a_{v, \text { wrist }}}{a_{v, \text { handlebar }}}
$$

The arithmetic means of transmissibility obtained in five replications were calculated. An analysis of variance (ANOVA) [7] was performed using the software Minitab 19 (Minitab LLC., State College, PA, USA). Since the hand equipment and hand size factors could have different effects for 
each load condition, three separate ANOVAs, with a level of significance of $95 \%(1-\alpha=0.95)$, were performed.

\section{Results}

Minitab provided $p$-values (Table 1) to understand the significance level of each factor in each load scenario. Table 1 displays that hand size did not affect vibrations transmission at all, while hand equipment had a significant effect just in the load condition $\mathrm{A}$, where the $p$-value was below the threshold value $\alpha=0.05$.

Table 1. $p$-values for each load condition.

\begin{tabular}{cccc}
\hline Load condition & $\begin{array}{c}\boldsymbol{p} \text {-Value } \\
\text { (for hand size) }\end{array}$ & $\begin{array}{c}\boldsymbol{p} \text {-Value } \\
\text { (for hand equipment) }\end{array}$ & $\begin{array}{c}\text { Threshold Value } \\
(\boldsymbol{\alpha})\end{array}$ \\
\hline $\mathrm{A}(\mathrm{d}=0.98 \mathrm{~mm} ; \mathrm{f}=20 \mathrm{~Hz})$ & 0.269 & 0.005 & 0.05 \\
$\mathrm{~B}(\mathrm{~d}=0.71 \mathrm{~mm} ; \mathrm{f}=40 \mathrm{~Hz})$ & 0.326 & 0.767 & 0.05 \\
$\mathrm{C}(\mathrm{d}=0.08 \mathrm{~mm} ; \mathrm{f}=90 \mathrm{~Hz})$ & 0.587 & 0.725 & 0.05 \\
\hline
\end{tabular}

Figure 3 shows the main effect plot for transmissibility of control factors hand size and hand equipment in load cycle A. Padded gloves gave the highest transmissibility value while the lowest one corresponded to the no gloves condition.

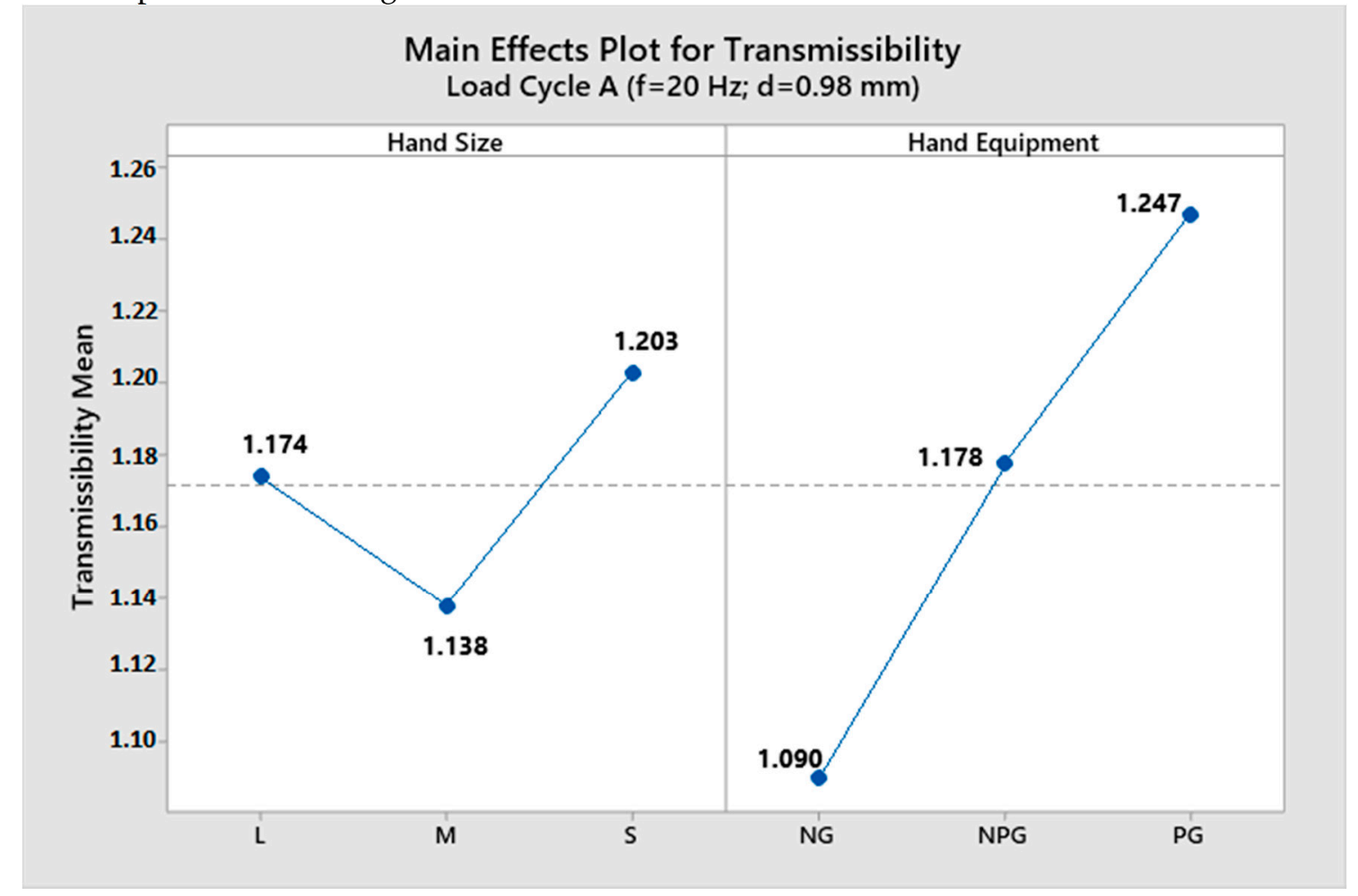

Figure 3. Main effects plot for transmissibility for load cycle A.

In Table 2, the arithmetic means and standard deviations of transmissibility are shown. On average, there was a minor increase in vibration transferred from handlebar to wrist (transmissibility $>1.0$ ) in load condition A (1.12). In load condition B, acceleration measured at the wrist was 1.82 times higher than on the handlebar. In load condition $C$ only, a reduced vibration was observed when transmissibility was averaged (0.56). 
Table 2. Arithmetic means and standard deviations for transmissibility of all six subjects.

\begin{tabular}{cccc}
\hline Load Condition & Hand Condition & Arithmetic Mean & Standard Deviation \\
\hline A & NG & 1.090 & 0.070 \\
A & NPG & 1.178 & 0.077 \\
A & PG & 1.247 & 0.057 \\
B & NG & 1.882 & 0.352 \\
B & NPG & 1.771 & 0.257 \\
B & PG & 1.800 & 0.191 \\
C & NG & 0.504 & 0.182 \\
C & NPG & 0.580 & 0.216 \\
C & PG & 0.610 & 0.272 \\
\hline
\end{tabular}

\section{Discussion}

By analyzing the cyclist's hand in the drops position, it was possible to note that the padded zones of the glove did not fully cover the contact area on the handlebar. This could explain why padded gloves had no positive effect on transmissibility. Further tests with different positions on the handlebar and especially with 'top' one, where padded zones completely cover the contact area, could show an effect. Even Drouet et al. [5], who used 'hoods' position for the test and gloves from a different brand, found that padded gloves had no significant effect and stated that 'top' position could better match padding layout with the cyclist grip on the handlebar.

The 'drops' position used in our tests was chosen with a pre-test that determined that the most severe, in terms of vibrations transmission, was the one on the brake levers. However, this one did not guarantee the same grip between all subjects, and hence lead to non-replicable tests and misleading results. The second most severe position was the one in the drops. This allowed subjects to hold the handlebar with all fingers. Moreover, this one was important because it is often used to perform descent.

The study of Laszlo and Griffin [4] on working gloves also showed no effect of hand size on vibrations transmission. Thus, further studies may not consider hand size as a control factor in the test design.

\section{Conclusions}

This study showed that cycling gloves had no significant effect on the reduction of vibrations coming from the road profile to the hand-arm system of a cyclist. The reason could be that cycling gloves were not designed to reduce vibrations and that the padding layout was not suitable for the position adopted in the test. Further studies should focus on the possibility of using a larger variety of gloves from different brands to analyze the effect of gloves when using different positions on the handlebar. In order to raise the comfort and the safety of the cyclist while using the position in the drops, new cycling gloves could be designed which pay close attention to the vibration's reduction.

Acknowledgments: G.S. traineeship was co-funded by the Erasmus+ Programme of the European Union. The activity is carried out in the international agreement program between Chemnitz University of Technology and University of Naples Federico II.

Conflicts of Interest: The authors declare no conflict of interest.

\section{References}

1. Mansfield, N.J. Human Response to Vibration; CRC Press: Boca Raton, FL, USA, 2005.

2. Lépine, J.; Champoux, Y.; Drouet, J.-M. Road bike comfort: On the measurement of vibrations induced to cyclist. Sports Eng. 2014, 17, 113-122. doi:10.1007/s12283-013-0145-8.

3. Olieman, M.; Marin-Perianu, R.; Marin-Perianu, M. Measurement of dynamic comfort in cycling using wireless acceleration sensors. Procedia Eng. 2012, 34, 568-573. doi:10.1016/j.proeng.2012.04.097. 
4. Laszlo, H.E.; Griffin, M.J. The transmission of vibration through gloves: effects of push force, vibration magnitude and inter-subject variability. Ergonomics 2011, 54, 488-496. doi:10.1080/00140139.2011.562984.

5. Drouet, J-M.; Covill, D.; Duarte, W. On the Exposure of Hands to Vibration in Road Cycling: An Assessment of the Effect of Gloves and Handlebar Tape. In Proceedings of the 12th Conference of the International Sports Engineering Association, Brisbane, QLD, Australia, 26-29 March 2018.

6. International Standards Organization. Mechanical Vibration-Measurement and Evaluation of Human Exposure to Hand-Transmitted Vibration - Part 1: General Requirements; ISO 5349-1; ISO: Geneva, Switzerland, 2001.

7. Montgomery, D.C.; Runger, G.C. Design of Experiments with Several Factors. In Applied Statistics and Probability for Engineers, 4th ed.; Wiley: Hoboken, NJ, USA, 2007; pp. 538-617.

(C) 2020 by the authors. Licensee MDPI, Basel, Switzerland. This article is an open access article distributed under the terms and conditions of the Creative Commons Attribution (CC BY) license (http://creativecommons.org/licenses/by/4.0/). 